\title{
Tonic-Clonic Seizure in a Liver-Transplant Recipient due to Concomitant Use of Colchicine with Cyclosporine A and Valganciclovir
}

\section{Iris Wai-Sum Li1 ${ }^{1,2 *}$ and Kenneth Siu-Ho Chok ${ }^{3,4}$ \\ ${ }^{1}$ Queen Mary Hospital, Hospital Authority, Hong Kong SAR, China}

${ }^{2}$ School of Public Health, Li Ka Shing Faculty of Medicine, The University of Hong

Kong, Hong Kong SAR, China

${ }^{3}$ Department of Surgery, The University of Hong Kong, Hong Kong SAR, China

${ }^{4}$ State Key Laboratory for Liver Research, The University of Hong Kong, Hong Kong SAR, China

*Corresponding author: Iris Wai-Sum Li, The University of Hong Kong, Queen Mary Hospital, 102 Pokfulam Road, Hong Kong SAR, China, Tel: +852-66822828; Email: irisli.irisli.irisli@gmail.com

\begin{abstract}
Use of immunosuppressant was inevitable in post-transplant recipients for prevention and treatment of allograft rejection. Concomitant use of antivirals in prevention of Cytomegalovirus disease after transplantation were common; and reduction in acute allograft rejection and all-cause mortality had also been shown. However, adverse effects from potential interactions between immunosuppressants and antiviral would have been overlooked easily; especially when adverse effects were further potentiated by addition of other medications for trivial conditions. Colchicine was commonly prescribed for clinical gout that occurred at higher incidences in post-transplant recipients on cyclosporine A or tacrolimus. We reported a post-LT patient developed his first tonic-clonic seizure from concomitant use of colchicine with CSA and valganciclovir.
\end{abstract}

Keywords: Seizure; Colchicine; Cyclosporine A; Valganciclovir; Liver transplant
Abbreviations: CMV: Cytomegalovirus; LT: Liver Transplantation; CSA: Cyclosporine A; MMF: Mycophenolate Mofetil; SOT: Solid-Organ Transplant; CSF: Cerebrospinal Fluid.

\section{Introduction}

Use of immunosuppressant was inevitable in posttransplant recipients for prevention and treatment of allograft rejection. Concomitant use of immunosuppressant and antivirals was common in transplant recipients [1]. Prevention of Cytomegalovirus (CMV) disease after liver transplantation (LT) by either pre-emptive therapy or antiviral prophylaxis, including ganciclovir (intravenous or oral) or valganciclovir, had been shown to reduce CMV infection by $40 \%$ and $\mathrm{CMV}$ disease by $58-80 \%$ respectively, and a $25 \%$ reduction in acute allograft rejection and all-cause mortality $[2,3]$. 


\section{Journal of Infectious Diseases \& Travel Medicine}

Hyperuricaemia and clinical gout had been noted in postLT patients on cyclosporine A (CSA) or tacrolimus [4]. Up to $6 \%$ and $28 \%$ of post-LT and post-transplant patients respectively developed clinical gout that colchicine might be required [5, 6]. However, such drug combinations in post-transplant recipients were not without risk, in particular the adverse effects from potential drug-drug interactions. We reported a post-LT patient developed his first tonic-clonic seizure from concomitant use of colchicine with CSA and valganciclovir; and subsequent recurrence even on CSA and valganciclovir without colchicine.

\section{Case}

The patient was a 62-year-old man who was a recipient of living-donor liver transplantation for acute liver failure with right anterior sectionectomy done for hepatitis B virus (HBV)-related hepatocellular carcinoma. He also had history of hypertension. He did not receive any preLT conditioning therapy and post-LT anti-rejection therapy. He had completed immediate-post-LT 3-months antiviral CMV prophylaxis. His immunosuppressant postLT included CSA and mycophenolate mofetil (MMF).

On day-158 post-LT, he was admitted with a 5-day history of productive cough, rhinorrhoea, malaise, low grade temperature and chills, abdominal pain and diarrhoea. His mental and hemodynamic status was stable. Chest auscultation and X-ray showed right lower zone coarse crepitations, and right lower zone haziness respectively. Initial investigations were performed (Table 1). Empirical antimicrobial therapy was started and treated as community-acquired pneumonia. CSA, as part of his usual immunosuppressant regimen was continued but MMF was stopped. His nasopharyngeal swab for viral culture was subsequently positive for Parainfluenza virus type 4 (Table 1).

\begin{tabular}{|c|c|c|}
\hline Tests & Results & $\begin{array}{c}\text { Reference } \\
\text { range }\end{array}$ \\
\hline $\begin{array}{c}\text { Complete blood } \\
\text { picture }\end{array}$ & & $3.7-9.3$ \\
\hline $\begin{array}{c}\text { White blood cell (wcc, } \\
\text { x109 } / \mathrm{L})\end{array}$ & 4.09 & $1.8-6.2$ \\
\hline Neutrophil $\left(\mathrm{x} 10^{9} / \mathrm{L}\right)$ & 3.71 & $1.0-3.2$ \\
\hline Lymphocyte $(\mathrm{x} \mathrm{109} / \mathrm{L})$ & 0.17 & $0.2-0.7$ \\
\hline Monocyte $\left(\mathrm{x} 10^{9} / \mathrm{L}\right)$ & 0.21 & $11.5-15.4$ \\
\hline Hemoglobin $(\mathrm{Hb}, \mathrm{g} / \mathrm{dL})$ & 8.5 & $8.1-11.5$ \\
\hline Platelet $\left(\mathrm{Plt}, \mathrm{x} 10^{9} / \mathrm{L}\right)$ & 127 & \\
\hline
\end{tabular}

Iris Wai-Sum Li and Kenneth Siu-Ho Chok. Tonic-Clonic Seizure in a Liver-Transplant Recipient due to Concomitant Use of Colchicine with Cyclosporine A and Valganciclovir. J Inf Dis Trav Med 2018, 2(1): 000108.

\begin{tabular}{|c|c|c|}
\hline \multicolumn{3}{|l|}{ Clotting profile } \\
\hline PT (sec) & 12 & $10.1-12.4$ \\
\hline INR & 1.1 & \\
\hline APTT (sec) & 30.3 & $25.1-35.5$ \\
\hline \multicolumn{3}{|l|}{ Renal function test } \\
\hline Sodium (Na, mmol/L) & 129 & $136-145$ \\
\hline Potassium (K, mmol/L) & 3.6 & $3.5-5.1$ \\
\hline Urea (mmol/L) & 18.2 & $<8.3$ \\
\hline Creatinine (umol/L) & 191 & $44-80$ \\
\hline \multicolumn{3}{|l|}{ Liver function test } \\
\hline Total protein $(\mathrm{g} / \mathrm{L})$ & 54 & $64-83$ \\
\hline Albumin (Alb, g/L) & 23 & $35-52$ \\
\hline Globulin (Glo, g/L) & 31 & $24-37$ \\
\hline Bilirubin, total $(\mu \mathrm{mol} / \mathrm{L})$ & 5 & $<17$ \\
\hline $\begin{array}{l}\text { Alkaline phosphatase } \\
\text { (ALP, U/L) }\end{array}$ & 45 & $35-104$ \\
\hline $\begin{array}{c}\text { Alanine transaminase } \\
\text { (ALT, U/L) }\end{array}$ & 21 & $<33$ \\
\hline $\begin{array}{c}\text { Aspartate transaminase } \\
\text { (AST, U/L) }\end{array}$ & 28 & $<38$ \\
\hline \multicolumn{3}{|l|}{$\begin{array}{l}\text { Nasopharyngeal } \\
\text { aspirate }\end{array}$} \\
\hline $\begin{array}{l}\text { Direct antigen detection } \\
\text { by immunofluorescence }\end{array}$ & \begin{tabular}{|c|} 
Negative for Influenzo \\
A \& B viruses, \\
Parainfluenza viruses \\
$1,2 \& 3$, Respiratory \\
Syncytial virus and \\
Adenovirus \\
\end{tabular} & \\
\hline Viral culture & $\begin{array}{c}\text { Positive for } \\
\text { Parainfluenza virus } 4\end{array}$ & \\
\hline
\end{tabular}

Table 1: Results of initial laboratory investigation for the patient.

On day-3 post-admission, he developed acute gouty attack and treated with oral colchicine $0.5 \mathrm{mg}$ twice daily. On day-5 post-admission, he was found to have CMV pp65 antigenemia, oral valganciclovir $450 \mathrm{mg}$ at 48 hourly was prescribed. On day-7 post-admission, the CMVpp65 antigen in plasma decreased from 30 per $2 \times 10^{5}$ white blood cells (wbc) to 8 per $2 \times 10^{5}$ wbc after 2-day valganciclovir use. After concomitant use of CSA, valganciclovir, and colchicine, he developed the first episode of tonic-clonic convulsion lasting $<1$ minute for 3 times, each of which was spontaneously aborted and subsequently controlled by phenytoin. CSA, valganciclovir, and colchicine were stopped immediately. Other medications including entecavir and ticarcillin-clavunate were continued (Table 2). Laboratory investigations were unremarkable with slightly improved urea and creatinine levels at 13 and $129 \mathrm{mmol} / \mathrm{L}$ respectively, normal glucose 
$4.3 \mathrm{mmol} / \mathrm{L}$ and ammonia <9umol/L. The magnetic resonance imaging of the brain showed cerebral microangiopathic and atrophic changes without features suggestive of encephalitis. Lumbar puncture was performed and the cerebrospinal fluid (CSF) analysis and microbiological investigations were unremarkable (Table 3).

\begin{tabular}{|c|c|}
\hline $\begin{array}{l}\text { Cerebrospinal fluid (CSF) } \\
\text { (reference range) }\end{array}$ & Result \\
\hline Colour & Colorless \\
\hline Turbidity & Clear \\
\hline Total cell count & 0 \\
\hline Erythrocyte (absent) & Absent \\
\hline Protein $(0.12-0.60 \mathrm{~g} / \mathrm{L})$ & 0.28 \\
\hline Glucose $(2.2-3.9 \mathrm{mmol} / \mathrm{L})$ & 3.9 \\
\hline Cryptococcal antigen (negative) & Negative \\
\hline \multicolumn{2}{|l|}{ Smear } \\
\hline Gram stain & Negative \\
\hline Acid-fast bacilli (AFB) & Negative \\
\hline Fungus & Negative \\
\hline \multicolumn{2}{|l|}{ Culture } \\
\hline Bacteria & No growth \\
\hline AFB & No growth \\
\hline Fungus & No growth \\
\hline \multicolumn{2}{|l|}{ PCR } \\
\hline Herpes simplex virus & Negative \\
\hline Varicella-zoster virus & Negative \\
\hline Enterovirus & Negative \\
\hline JC virus & Negative \\
\hline Mycobacterium tuberculosis complex & Negative \\
\hline \multicolumn{2}{|l|}{ Blood } \\
\hline Glucose $(2 \mathrm{hr} \mathrm{pp}<7.8 \mathrm{mmol} / \mathrm{L})$ & 5.6 \\
\hline Bacterial culture & No growth \\
\hline \multicolumn{2}{|l|}{ Mid-stream urine } \\
\hline Bacterial culture & No growth \\
\hline
\end{tabular}

Table 3: Results of biochemical and microbiological investigations performed for the first seizure of our patient.

He developed another episode of tonic-clonic convulsion at 5-days after reintroduction of CSA and valganciclovir even on phenytoin, without concomitant use of colchicine. Both CSA and valganciclovir were then stopped immediately. The serum CSA drug level profile had been within reference range all-along. CSA was subsequently reintroduced alone, and no seizure for $\geq 6$ months during CSA monotherapy as immunosuppressant, and remained seizure-free on subsequent concomitant use of sirolimus and prednisolone.

\section{Discussion}

Seizure was the second most common neurologic complications post-LT [7, 8], with reported incidence varied from $15 \%$ to $40 \%$ [7-9], and a higher incidence up to $33 \%$ in re-LT recipients [9]. Our patient's seizure developed at $\sim 22$ weeks post-LT, which was later than the typically described period: $>2 / 3$ developed in the initial 4 weeks early post-LT or in bimodal distribution that $>50 \%$ occurred in the initial week, and then 5 to 16 weeks post-LT [7-9]. Concordant with previous reports, tonic-clonic seizure was the commonest form of seizure in post-LT recipients [7-9]. About $9 \%$ to $12 \%$ of seizures in post-LT recipients were status epilepticus [7, 9]. The cause of seizure could be multifactorial. Causes such as electrolyte and metabolic disturbances, central nervous system infection and structural abnormalities, hypoxicischaemic encephalopathy, cerebrovascular complications [7-10] had been excluded in our patient.

Parainfluenza virus type 4 was isolated from our patient's nasopharyngeal aspirate. However, his clinical, CSF and radiological findings were not suggestive of viral encephalitis and/or post-infectious encephalitis; the latter was considered as immune-mediated usually occur at 2 to 30 days after initial infection [11].

Our patient's seizure was unlikely solely due to CSA, as part of CSA acute encephalopathy and seizure syndrome, or CSA neurotoxicity. The latter would also present with tremors, altered conscious state and encephalopathy, which occurred at $1 \%$ to $10 \%$ or relatively higher in solidorgan transplant (SOT) patients; and mediated through inhibition of $\gamma$-aminobutyric acid (GABA)-ergic transmission and activation of serotoninergic neural activities [12-14]. Our patient had been seizure-free allalong on CSA, either in combination with MMF before admission, or subsequent monotherapy after his last seizure episode, or later concomitantly with sirolimus and prednisolone. Moreover, he did not experience any common side effects of CSA, such as exaggerated blood pressure on his underlying hypertension, gingival hyperplasia, hirsutism, and mild-to-moderate hyperbilirubinemia [15]. Nevertheless, our patient's serum CSA levels were concordant with previous findings that serum trough CSA levels were found within reference range in seizures of post-LT patients, despite neurotoxicity usually occur when higher doses were used 
[10]. Mice model with partial hepatectomy in simulation to transient liver dysfunction at early phase post-LT was shown to increase susceptibility of CSA-induced neurotoxicity mediated by reduced blood-brain barrier function [13]. This might explain seizure occurrence in early weeks post-LT. CSA, a calcineurin inhibitor, had been widely used to prevent allograft rejection in SOT patients and to treat various autoimmune diseases by inhibit the production and release of interleukin-2 (IL-2), and the IL-2-induced activation of resting T-lymphocytes [12].

Concomitant use of CSA with colchicine increased the colchicine maximum observed plasma concentration which might potentiate colchicine gastrointestinal side effects, dose-related myelosuppression, and other toxicities such as renal and liver impairment, polyneuropathy, myopathy, rhabdomyolysis and cardiovascular effects such as heart failure and ventricular arrhythmia $[15,16]$. Nevertheless, these were not evident in our patient. CSA had been shown to inhibit colchicine excretion via kidney and liver in animal model [15]. Moreover, CSA also inhibit colchicine efflux from nerve and muscle cells resulted in neurotoxicity and myotoxicity especially in renal and liver impaired patients [16].

The mechanism was due to potent inhibitory effect of CSA on P-glycoprotein drug transport system [16]. It was suggested that either a $50 \%$ reduced colchicine dose was used whenever with CSA was concomitantly indicated, or a switch of CSA to other immunosuppressants; and similar was applicable to tacrolimus [16]. Other immunosuppressant such as FK506 and OKT3 also predisposed to seizure development [10]. Our patient received colchicine at $0.5 \mathrm{mg}$ twice daily, which seems to be higher than the studied dose of $0.6 \mathrm{mg}$ daily and the suggested reduced dose at $0.3 \mathrm{mg}$ daily orally [16]. On the other hand, colchicine had been reported as a convulsant agent in animal models $[17,18]$. Colchicine, which was a tubulin depolymerizing agents, its neurotoxicity had been shown related to destruction of microtubules of selected neural cells leading to accumulation of metabolic toxic cellular products [17]. Colchicine was shown to induce hippocampal granule cells hyperexcitability with interictal epileptic spike or electrographic seizures [18]. Colchicine was shown to be competitive antagonist at human recombinant $\gamma$-GABA(A) receptor, either through direct interaction at receptor binding site or receptor affinity modulation by allosteric interaction with other binding site or receptor associated protein; and CSA was shown to enhance colchicine induced apoptosis in cerebellar granule neurons of rats [19]. Concomitantly, CSA absorption was increased by concomitant use of colchicine, hence the CSA drug level in blood [12]. Transplant or autoimmune-diseased patients on CSA were found to have a higher incidence of hyperuricemia and clinically gouty attack $[16,20]$. When colchicine was prescribed for the latter, this might initiate drug interactions for complications.

An apparent temporal relationship to the concomitant introduction of CSA and valganciclovir, with and without colchicine, was observed for the seizure recurrence in our patient. Drug interactions between immunosuppressant and antiviral with tonic-clonic convulsion in patients with recurrent herpetic keratitis [11], transient neutropenia and its association of trough MMF level with valganciclovir in renal-transplant patients [16], had been suggested previously for MMF but not for CSA. MMF in our patient had been stopped before his first seizure developed, despite its metabolite mycophenolic glucuronide would raise the intracellular concentration of acyclovir or valganciclovir to haematotoxic levels by competing for renal tubular secretion, resulted in exacerbation of neurotoxicity and cytopenic side effects of acyclovir [21,22] However, valganciclovir had been shown causing additive nephrotoxicity with CSA [23]. Valganciclovir had been widely used for prevention of CMV infection in high-risk SOT recipients, with good tolerability except the higher incidences of neutropenia that usually developed after prolonged use for 3 months, and nephrotoxicity $[2,24,25]$. Tonic-clonic convulsion was rarely occurred at $<5 \%$ as side effect of valganciclovir [26]. The neurotoxicity of valganciclovir was reported in $<1 \%$ of adult post-LT patients on low dose as prophylaxis; and manifested as mental confusion and hallucination in a child post-haemopoietic stem cell transplant without concomitant CSA use $[27,28]$.

\begin{tabular}{|c|}
\hline Cyclosporine A \\
\hline Valganciclovir \\
\hline Colchicine \\
\hline Entecavir \\
\hline Ticarcillin-clavunate \\
\hline Fluconazole \\
\hline Metoprolol \\
\hline Amlodipine \\
\hline Esomeprazole \\
\hline Ursodeoxycholic acid \\
\hline
\end{tabular}

Table 2: List of medications the patient was taken when the first seizure developed.
Iris Wai-Sum Li and Kenneth Siu-Ho Chok. Tonic-Clonic Seizure in a Liver-Transplant Recipient due to Concomitant Use of Colchicine with Cyclosporine A and Valganciclovir. J Inf Dis Trav Med 2018, 2(1): 000108.
Copyright $($ Iris Wai-Sum Li and Kenneth Siu-Ho Chok. 
In summary, we reported a rarely occurred complication from a commonly used drug combination regimen, which not only for post-LT, but also other posttransplant patients in clinical practice. Vigilant awareness from clinicians and transplant expertise were important to note such potentially fatal complication or with significant morbidity from drug combination and interaction. Further researches were needed for underlying mechanisms of the drug interaction, so as to guide further for clinical recommendations accordingly.

\section{References}

1. Asberg A, Jardine AG, Bignamini A, Rollag H, Pescovitz MD, et al. (2010) Hartmann A on behalf of the VICTOR Study Group. Effects of the intensity of immunosuppressive therapy on outcome of treatment for CMV disease in organ transplant recipients. Am J Transplant 10(8): 1881-1888.

2. Dummer JS (1990) Cytomegalovirus infection after liver transplantation: clinical manifestations and strategies for prevention. Rev Infect Dis 12 (7): S767775.

3. Andrews PA, Emery VC, Newstead C (2011) Summary of the British Transplantation Society Guidelines for the Prevention and Management of CMV Disease After Solid Organ Transplantation. Transplantation 92(11): 1181-1187.

4. Gerster J, Dudler M, Halkic N (2004) Gout in liver transplant patients receiving tacrolimus. Ann Rheum Dis 63(7): 894-895.

5. Neal DA, Tom BD, Gimson AE, Gibbs P (2001) Hyperuricemia, gout, and renal function after liver transplantation. Transplantation 72(10): 1689-1691.

6. Stamp L, Searle M, O'Donnell J, Chapman P (2005) Gout in solid organ transplantation: a challenging clinical problem. Drugs 65(18): 2593-2611.

7. Derle E, Kibaroğlu S, Öcal R, Kırnap M (2015) Seizure as a neurologic complication after liver transplant. Exp Clin Transplant 13 (Suppl 1): 323-326.

8. Bronster DJ, Emre S, Mor E, Sheiner P (1994) Neurologic Complications of Orthotopic Liver Transplantation. Mt Sinai J Med 61(1): 63-69.

Iris Wai-Sum Li and Kenneth Siu-Ho Chok. Tonic-Clonic Seizure in a Liver-Transplant Recipient due to Concomitant Use of Colchicine with Cyclosporine A and Valganciclovir. J Inf Dis Trav Med 2018, 2(1): 000108.
9. Wszolek ZK, Steg RE (1997) Seizure after orthotopic liver transplantation. Seizure 6(1): 31-39.

10. Wszolek ZK, Steg RE (1995) Seizures after liver transplantation. Liver transplantation 1(5): 334-339.

11. Sonneville R, Klein I, de Broucker T (2009) Postinfectious encephalitis in adults: diagnosis and management. J Infect 58(5): 321-328.

12. (2017) Cyclosporin (ciclosporin) (systemic): drug information. Lexicomp® 1978-2017. Lexicomp, Inc.

13. Yamauchi A, Dohgu S, Takata F, Watanabe T, Nishioku $\mathrm{T}$, et al. (2011) Partial hepatectomy aggravates cyclosporine A-induced neurotoxocity by lowering the function of blood-brain barrier in mice. Life Sci 88(11-12): 529-534.

14. Moorman MT, Epstein RB, Smith JW, O'Neal C (2011) Management of cyclosporine overdose in a hematopoietic stem cell transplant patient with sequential plasma exchange and red blood cell exchange. J Clin Apher 26(3): 156-158.

15. Wason S, Digiacinto JL, Davis MW (2012) Effect of cyclosporine on the pharmacokinetics of colchicines in healthy subjects. Postgrad Med 124(4): 189-196.

16. Eleftheriou G, Bacis G, Fiocchi R, Sebastiano R (2008) Colchicine-induced toxicity in a heart transplant patient with chronic renal failure. Clin Toxicol (Phila) 46(9): 827-830.

17. Steward O, Goldschmidt RB, Sutula T (1984) Neurotoxicity of colchicine and other tubulin-binding agents: a selective vulnerability of certain neurons to the disruption of microtubules. Life Sci 35(1): 43-51.

18. Sutula T, Goldschmidt R, Steward O (1983) Mechanisms of colchicine neurotoxicity in the dentate gyrus: dissociation of seizures and cell death. Exp Neurol 81(3): 683-693.

19. Weiner JL, Buhler AV, Whatley VJ, Harris RA (1998) Colchicine is a competitive antagonist at human recombinant gamma-aminobutyric acid A receptors. J Pharmacol Exp Ther 284(1): 95-102.

20. Clive DM (2000) Renal transplant-associated hyperuricemia and gout. J Am Soc Nephrol 11(5): 974-979. 
21. Lee KM, Kim MK, Wee WR, Lee JH (2011) Tonic-clonic seizure following combined treatment of mycophenolate mofetil and acyclovir. Graefes Arch Clin Exp Ophthalmol 249(7): 1107-1108.

22. Royer B, Zanetta G, Bérard M, Davani S, Tanter Y, et al. (2003) A neutropenia suggesting an interaction between valacyclovir and mycophenolate mofetil. Clin Transplant 17(2): 158-1561.

23. Valganciclovir Film Coated Tablets Product Monograph, 05 December 2017.

24. Lee SO, Razonable RR (2010) Current concepts on cytomegalovirus infection after liver transplantation. World J Hepatol 2(9): 325-336

25. Said T, Nampoory MRN, Pacsa AS, Essa S, Madi N, et al. (2007) Oral valganciclovir versus intravenous gancyclovir for cytomegalovirus prophylaxis in kidney transplant recipients. Transplant Proc 39(4): 997-999.

26. (2017) Valganciclovir: drug information. Lexicomp® 1978-2017 Lexicomp, Inc.

27. Dupuis R, Harris M, Gillis K, Gerber D (2007) Experience with low-dose valganciclovir prophylaxis in adult liver transplant recipients. Transplant Proc 39(10): 3266-3270.

28. Peyrière H, Jeziorsky E, Jalabert A, Cociglio M (2006) Neurotoxicity related to valganciclovir in a child with impaired renal function: usefulness of therapeutic drug monitoring. Ann Pharmacother 40(1): 143-146. 